\title{
Enhanced Segmentation Procedure for Intima- Adventitial Layers of Common Carotid Artery
}

\author{
V.Savithri ${ }^{1}$ and S.Purushothaman ${ }^{2}$ \\ ${ }^{1}$ Department of Computer Science, Mother Teresa Women's University, Kodaikanal,India \\ ${ }^{2}$ Principal, Sun College of Engineering and Technology, Kanyakumari, India \\ Email: savi3_8@yahoo.co.in,dr.purushothaman.s@gmail.com
}

\begin{abstract}
This paper presents an enhanced Segmentation technique for use on noisy B-mode ultrasound images of the carotid artery. This method is based on Image Enhancement, Edge detection and Morphological operations in boundary detection. This procedure may simplify the job of the practitioner for analyzing accuracy and variability of segmentation results. Possible plaque regions are also highlighted. A thorough evaluation of the method in the clinical environment shows that inter observer variability is evidently decreased and so is the overall analysis time. The results demonstrate that it has the potential to perform qualitatively better than applying existing methods in intima and adventitial layer detection on $B$-mode images.
\end{abstract}

Keywords - Artery, boundary detection, imaging, Ultrasonic, parallel programming

\section{INTRODUCTION}

According to Madras Medical College, stroke disease costs India more than 1100 billion rupees per year, and this value continues to increase with each passing year [1]. This amount covers the cost of care and research to help alleviate the dreadful effects of this disease. In order to help such costs, techniques to help simplify and reduce a medical practitioner's daily workload need to be developed.

Accurate measurement and understanding of the geometry of carotid arteries are important in the assessment and management of risk for stroke. An indication of early symptoms, which can be used as a pre-clinical disease marker, is a thickening of carotid plaque levels may block the passage of blood traveling to the upper body and brain. However, plaque and boundary identification in ultrasound images is still a tedious and difficult task due to lack of its automation. Currently in India, practitioners identify plaque regions and other myocardial tissues in ultrasound images by manually tracing their borders to best fit the data.

Several studies are being conducted to improve the quality and detection within ultrasound images [2] designed an efficient dynamic programming algorithm to detect boundaries of carotid artery. Their algorithm creates an accurate boundary detection.[3] designed a dual boundaries with the optimal solution based on a given cost function. However, their algorithms may not give depth study of vessel detection in far-wall adventitia. The basic research on the feasibility study of using B-mode sonographic images to measure the arterial IMT is reported in [20]. Afterwards, some related problems such as measuring the arterial elasticity have been studied [4],[15],[16]. Also, the automatic measurement of IMT variation and lumen diameter variation over cardiac cycles have been investigated [12]. Early studies of automatic detection on static B-mode images can be found in [6] and
[17]-[19]. However, they have been performed without any anatomic knowledge on intima and adventitia. Dynamic programming (DP) approaches[13] [21],[22] have been employed to solve many image analysis and computer vision problems such as stereo matching[23], contour detection [24],[25], contour tracking [25], and medical applications [18],[26],[27]. The DP method used for IMT measurement is known at least since 1994 [7]. The advantage of DP techniques is that optimal solution can be guaranteed. Generally, dynamic programming can handle primitive knowledge such as image features and curve smoothness. Seldom researchers [27],[29] take the structural knowledge into consideration.

The authors in [27],[14] proposed a sectional dynamic programming that can detect quasi-parallel lines such as the spline boundaries. [5], The proposed method is piecewise linear and is operated with some segments with a fixed distance defining the width of the spinal cord.[8] The authors treat the structural knowledge as geometric and consider hard constraints that the dynamic programming has to satisfy. Their method is specifically applicable to the spine boundaries. However, some applications require detection of curves with exact positions in pixel such as intima and adventitia detection of CCA wall of B-mode sonographic images [9],[10],[19]. Under [11] this requirement, the constraints might fail on this pixel scale. We illustrate this point by an example: the normal IMT of a normal adult is about $0.5 \mathrm{~mm}$. If the pixel size such as that of a Toshiba SSA-'380-A' scanner(Japan) is $0.096 \mathrm{~mm}$, then one pixel error results in about $20 \%$ error in a normal patient, which is unacceptable in clinical measurements.

The study in [29] proposed a method that takes the pixel scale into consideration in the multi dynamic programming. In order to reduce the computation time, some disjoint ness, ordering, spacing and heuristic constraints were suggested. The disjoint ness and spacing constraints can not only reduce the computation time but also help to satisfy the geometric constraints that are suitable for our application.

In this paper, a new technique is implemented to help identify carotid regions semi-automatically by segmenting ultrasound images. This technique would dramatically decrease the time needed to analyze the ultrasonic images. In order to accomplish this segmentation solution, image processing technique such as image enhancement, image threshold, image denoising, edge detection, boundary detection and segmentation, have been used to manipulate the image data.

\section{METHODOLOGY}




\section{Problem Definition}

The noise created during ultrasound scanning leads to difficulty in defining the boundary of the vessel. The image is further deteriorated by the occurrence of lipid rich plaque a poorly angled transducer during image acquisition. Difficult in highlighting plaque region.

\section{A. Enhanced Segmentation Procedure}

\section{Image Enhancement}

The goal of image enhancement is to improve the perception of the image through modification of intensity functions, image spectral content, or a combination of these functions. Removing blurring and noise, increasing contrast, and revealing details are examples of image-enhancement operations. The histogram of an image gives the frequency of occurrence of the gray levels.

Procedure to perform Histogram equalization

Step 1: Find the running sum of the histogram values.

Step 2: Normalise the values from step (1) by dividing by the total number of pixels.

Step 3: Multiply the values from step (2) by the maximum gray-level value and round.

Step 4 : Map the gray level values to the results from step (3) using a one-to-one correspondence.

\section{Image Thresholding}

Thresholding is a part of a more general segmentation problem. Thresholding is required to extract a part of an image which contains all the information. Thresholding is useful technique for establishing boundaries in images that 22 contain solid objects resting on a contrasting background. In hard thresholding, pixels having intensity lower than the threshold $\mathrm{T}$ are set to zero and the pixels having intensity greater than the threshold are set to 255 or left at their original intensity depending on the effect that is required. This type of hard thresholding allows us to obtain a binary image from a grayscale image. The mathematical expressions are:

The derivation of the area functions for an object whose boundary is defined by thresholding:

$$
\mathrm{H}(\mathrm{D})=-\mathrm{d} / \mathrm{dD} \mathrm{A}(\mathrm{D})
$$

Where D- gray level, A(D) - area of the object at gray level D, and $H(D)$ refers to Histogram.

III. Image Denoising

An image is often corrupted by noise in its acquisition or transmission. Noise is any undesired information that contaminates an image. Noise appears in images from a variety of sources. The goal of denoising is to remove the noise while retaining as much as possible the important signal features. Denoising can be done through filtering. Filters reduce noise. Gaussian highpass filter helps to reduces a noise.

$$
\mathrm{H}_{\mathrm{hfe}}(\mathrm{u}, \mathrm{v})=\mathrm{a}+\mathrm{b}_{\mathrm{Hhp}}(\mathrm{u}, \mathrm{v})
$$

Where a is the offset, $b$ is the multiplier, and $\mathrm{H}_{\mathrm{hp}}(\mathrm{u}, \mathrm{v})$ is the transfer function of the highpass filter.

\section{Edge Detection}

This procedure defines edges as Zero-crossings of second derivatives in the direction of the greatest first derivate. This works in multistage process (i) image is smoothed by Gaussian convolution (ii) 2D first derivate operator is applied to the smoothed image to highlight region of the image with high spatial derivatives. The effectiveness of this algorithm is determined by three parameters (i) width of the Gaussian kernel (ii) upper threshold (iii) lower threshold used by tracker.

$$
[\mathrm{g}, \mathrm{t}]=\text { edge (f, 'canny', } \mathrm{T}, \text { sigma) }
$$

Where $\mathrm{T}$ is a vector, $\mathrm{T}=[\mathrm{t} 1, \mathrm{t} 2]$, containing the two thresholds and sigma is the standard deviation of the smoothing filter.

V. Morphological operations for Boundary detection Morphological operations are very effective in the detection of boundaries in a binary image $X$. The following boundary detectors are widely used:

$$
\begin{gathered}
Y=X-(X \theta B) \\
Y=(X \circlearrowleft B)=X \text { or } \\
Y=(X \circlearrowleft B)-\left(\begin{array}{lll}
X & \theta & B
\end{array}\right)
\end{gathered}
$$

where $\mathrm{Y}$ is the boundary image, operator $\theta$ denotes erosion operator $\Theta$ denotes dilation ' - ' denotes set theoretical subtraction.

\section{$\%$ Boundary detector}

Close all;

Clear all;

Clc;

$\mathrm{a}=$ imread('carotid.jpg');

$\mathrm{b}=[010 ; 111 ; 010]$;

a1=imdilate $(a, b)$;

a2=imerode $(a, b)$;

$\mathrm{a} 3=\mathrm{a}-\mathrm{a} 2$;

$\mathrm{a} 4=\mathrm{a} 1-\mathrm{a}$

a5=a1-a2;

imshow(a)

figure,imshow(a1),title('Dilated Image')

figure, imshow(a2), title('Eroded Image')

\section{Segmentation of the intima-media region}

We introduce a split and merge method for the segmentation of the imtima-media region in ultrasound images, which combines splines(for the adventitia detection), dynamic programming(dp), smooth intensity thresholding surfaces and a successful geometric active contour model and known for its accuracy, flexibility and robustness. Several image features are used in the segmentation. Human interaction is minimal. 
It is able to segment both near-end and far-end carotid walls; it supports to detect plaques of different sizes, shapes and classes.

The basic steps of splitting and merging images are

Split into four disjoint quadrants any region Ri for which $\mathrm{P}\left(\mathrm{R}_{\mathrm{i}}\right)=$ FALSE

When no further splitting is possible, merge any adjacent regions $R_{j}$ and $R_{k}$ for which

$\mathrm{P}\left(\mathrm{R}_{\mathrm{j}} \mathrm{U} \mathrm{R} \mathrm{R}_{\mathrm{k}}\right)=$ TRUE.

Stop when no further merging is possible.

\section{RESULTS}

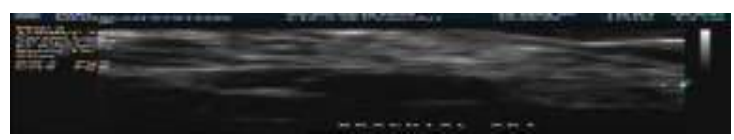

Original Image

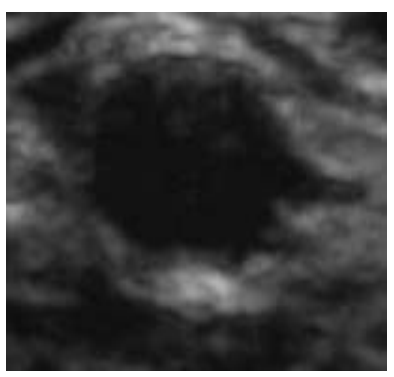

gray

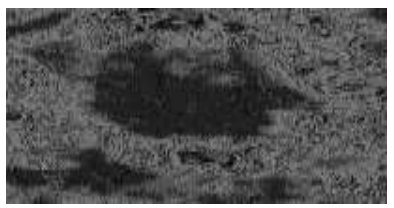

indexed

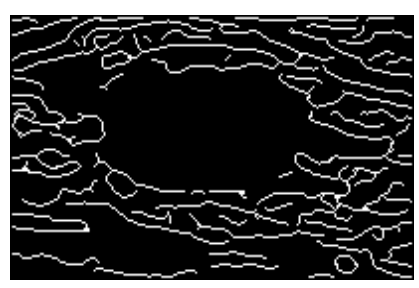

morphed

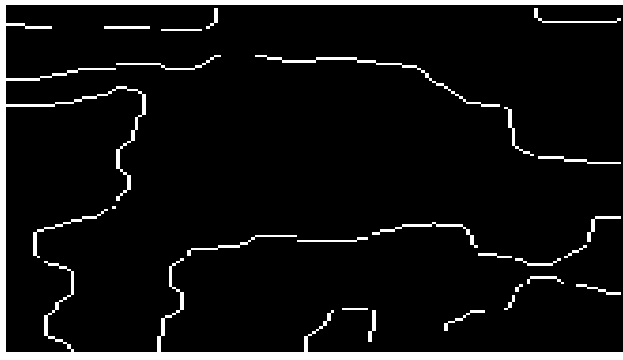

Segmentation

\section{DISCUSSION}

We have proposed a enhanced segmentation technique for detecting intima and adventitia in B-mode ultrasonic images. Our method inherits the property of guaranteeing the global minimal result from TDP. Simultaneously it increases the robustness against the speckle noise effect in the ultrasonic images. Our experimental results show that this method can effectively alleviate the problems caused by the TDP when applied in CCA(far-wall) IMT measurement. In general, Our segmentation technique demonstrates its superior performance over the TDP in this application

Table-1

Reading Variability (\%) when measurements were Performed by three readers before applying Procedure.

\begin{tabular}{|l|l|l|l|}
\hline $\begin{array}{l}\text { Reader1 } \\
\text { (Accurac } \\
\text { y) }\end{array}$ & $\begin{array}{l}\text { Reader2 } \\
\text { (Accuracy) }\end{array}$ & $\begin{array}{l}\text { Reader3 } \\
\text { (Accuracy) }\end{array}$ & $\begin{array}{l}\text { Average( } \\
\%) \\
\text { (Accuracy } \\
)\end{array}$ \\
\hline $82 \%$ & $80 \%$ & $84 \%$ & $82 \%$ \\
\hline
\end{tabular}

Table-2

Reading Variability (\%) when measurements were Performed by three readers after applying Procedure.

\begin{tabular}{|l|l|l|l|}
\hline $\begin{array}{l}\text { Reader1 } \\
\text { (Accuracy) }\end{array}$ & $\begin{array}{l}\text { Reader2 } \\
\text { (Accuracy) }\end{array}$ & $\begin{array}{l}\text { Reader3 } \\
\text { (Accuracy) }\end{array}$ & $\begin{array}{l}\text { Average(\% } \\
\text { (Accuracy) }\end{array}$ \\
\hline $92 \%$ & $92.5 \%$ & $94 \%$ & $93 \%$ \\
\hline
\end{tabular}

Our future aim is to test this system on images made by some other sonographic instrumentation. The system should be able to select features fully automatically according to different instrumentations, The final goal is to make this system portable within the current B-mode ultrasonic instrumentation. As a final remark, we want to point out that in addition to the intima and adventitia detection, several other medical applications are based on detecting near-parallel contours. The technique proposed in this paper is potentially applicable in these situations as well.

\section{CONCLUSION}

A method is proposed based on Enhanced segmentation procedure to automatically measure ultrasonic artery images. The human 
knowledge of the artery image is incorporated in the system, which makes the system capable in processing images of different quality. Human factors in the determination of the boundaries are reduced Evaluation of the system shows reduced inter observer variability as well as overall analysis time. The automated artery boundary detection and segmentation, system can replace the old manual system in a clinical application environment.

\section{ACKNOWLEDGMENT}

We would like to thank members of Madras Medical College and New hope Scan centre and Hospital, in the Department of Radiologist and Neurologist, offered valuable comments advices and for providing patients personal reports

\section{REFERENCES}

[1] Heart and Stroke Foundation, Annual report Findings, 2008

[2] Q.Liang, I.Wendelhag,J.wikstrand, and T.Gustavasson "A multiscale dynamic programming procedure for boundary detection in ultrasonic artery images,"IEEE Trans.Med.imag.vol.19,No.2,pp 127-142,feb.2000.

[3] Da-Chuan Cheng, Member,IEEE, and Xiaoyi Jiang, member, IEEE, "Detections of Arterial wall in Sonographic Artery images using Dual Dynamic Programming," IEEE TransMed Imag. Vol.12, No.6, November 2008.

[4] L. Angrisani, P. Daponte, C. Liguori, and A. Pietrosanto, "An image based measurement system for the characterization of automotive gaskets," Measurement, vol. 25, pp. 169-181, 1999.

[5] M. G. Bond and S. K. Wilmoth et al., "Detection and Monitoring of Asymptomatic Atherosclerosisi in Clinical Trials," Amer. J. Med., vol.86, (suppl 4A), pp. 33-36, 1989.

[6] N. M. El-Barghouty, T. Levine, S. Ladva, A. Flanagan, and A. Nicoladeis, "Histological verification of computerized carotid plaque characterization," Eur. J. Vascular Endovascular Surg., vol. 11, pp. 414-416, 1996.

[7] F. De Man, I. De Scheerder, M.C. Herregods, J. Piessens and H. De Geest Role of Intravascular Ultrasound in Coronary Artery Disease: A new gold standart? Beyond Angiography. Intravascular Ultrasound State-Of-The-Art XX Congres of the ESC, Vol 1 (August 1998)

[8] D. Hausmann, Andre J.S. Lundkvist, Guy Friedrich, Krishnankutty Sudhir, Peter J. Fitzgerald and Paul G. Yock Lumen and Plaque Shape in Atherosclerotic Coronary Arteries Assesed by In EVO Intracoronary Ultrasound Beyond Angiography. Intravascular Ultrasound: State-OfThe-Art XX Congres of the ESC, Vol 1 (August 1998)

[9] F. Escolano, M. Cazorla, D. Gallardo and R. Rizo Deformable Templates for Plaque Thickness Estimation of Intravascular Ultrasound Sequences Pattern Recognition and Image Analysis. Preprints of the VI1 National Symp. On Patt. Recog. and Im. An.Vol 1 (April 1997)

[10] M.A.Bottalico, A.Starita, "EcoStudio:A computer tool to support carotid ultrasound images analysis,Engineering in Medicine and Biology Soc.,IEEE,pp.2428-2430,2000.

[11] Song Chun Zhu, Alan Yuille, Region Competition:Unzfiin Snakes, Region Growing, and BayesIMDL for Multiband Image Segmentation. IEEE Trans. Pattern An. Mach. Intelligence, Vol. 18, No 9, ( September 1996).

[12] Nobuyuki Otsu A Threshold Selection Method from GrayLevel Histograms. IEEE Trans. on Sys. Man and Cybernetics, Vol. SMC-9,Na 1, pp 62-65, (January 1979)
[13] I. Wendelhag, T. Gustavsson, M. Suurkula, G. Berglund, and J. Wikstrand, "Ultrasound measurement of wall thickness in the carotid artery. Fundamental principles and description of a computerized analyzing system," Clin. Physiol., vol. 6, no. 11, pp. 565-577, 1991.

[14] P. Pignoli, E. Tremoli, A. Poli, and R. Paoletti, "Intimal plus medial thickness of the arterial wall: A direct measurement with ultrasound imaging," Circulation, vol. 74, pp. 13991406, 1986.

[15] R. E. Bellman and S. Dreyfus, Appled Dynamic Programming. Princeton, NJ: Princeton University Press, 1962.

[16] A. A. Amini, T. E.Weymouth, and R. C. Jain, "Using dynamic programming for solving variational problems in vision," IEEE Trans. Pattern Anal. Machine Intell., vol. 12, pp. 855-867, Sept. 1990.

[17] R. J. Kozick, "Detectinh interfaces on ultrasound images of the carotid artery by dynamic programming," SPIE, vol. 2666, pp. 233-241, 1996.

[18] W. Liang, R. Briwning, R. Lauer, and M. Sonka, "Automated analysis of brachial ultrasound time series," in Proc. SPIE Conf. Physiol. Function Multidimensional Images, vol. SPIE 3337, San Diego, CA, Feb. 1998, pp. 108-118.

[19] P.Pignoli,E.Tremoli,A.Poli,P.Oreste, and R.Paoletti, "Intimal plus medical thickness of the arterial wall: A direct measurement with ultrasound imaging", circulation, vol.74 pp. 1399-1406, 1986.

[20] L.Scharf and H.Elliott,"Aspects of dynamic programming in signal and image processing",IEEE, Trans Autom.control,Vol.AC-26,no.5,pp.1018-1029,Oct.1981.

[21] H.Burkhardt and H.Schorb,"Maximum-a-posteriori restoration of images-an application of the viterbi algorithm to two-dimenstional filtering" in Proc.Int.Conf.Pattern Recog.(ICPR).oct.,1982,pp.98-101.

[22] I.Cox S.Hingorani,S.Rao,B.Maggs,"A maximum likehood stereo algorithm", Computer Vs image understanding,vol.63,pp.24-45,1996.

[23] A.Amini,T.Weymouth, and R.Jain,"Using dynamic programming for solbving variational problems in vision", IEEE Trans.Pattern Analy.Mach. Intell.vol.12,no.9,pp 855867,sep. 1990 .

[24] D.Geiger,A.Gupta,L.A.CostaandJ.Vlontzos,'Dynamic programming for detecting,tracking matchingdeformablecontours,'IEEETrans.Pattern.Anal .Mach. Intell, vol. 17, no.3, pp.294302, mar.1995.

[25] Y.chen,T.Hung and Y.Rui,"optimal radial contour Tracking by dynamic programming, "in proc. Int. Conf .Image Process. (ICIP), 2001 pp.626-629.

[26] G.Wei,J.Qian, and H.Schramm, "Generalized Dynamic programming approaches for object detection:Detecting spine boundaries and vertebra endplates" in Proc.IEEE Conf. Comput.Vis.Pattern Recognis.(CVPR),2001 vol.1,pp.954-959.

[27] G.Aboutanos, J.Nikanna N.Watkins B.Dawant, Model creatinn and deformation the automatic segmentation of the brain in MR images, IEEE,

Trans.Biomed.Eng.vol.46,no.11,pp.1346-1356,nov.1999.

[28] C.Su and B.Appleton, "Multiple paths extraction in images using aconstrained expanded Trellis", IEEE Trans. Pattern Anal.Mach. Intell.,vol.27,no.12,pp.1923-

[29] L.MacLeod, "Comments on techniques for edge detection,"Proc.IEEE,vol.60,no.3,p.344,Mar.1972. 